\title{
OBJECT RECOGNITION IN PROBABILISTIC 3-D VOLUMETRIC SCENES
}

\author{
Maria I. Restrepo ${ }^{1}$, Brandon A. Mayer ${ }^{1}$ and Joseph L. Mundy ${ }^{2}$ \\ ${ }^{1}$ Ph.D. Student, School of Engineering, Brown University, 182 Hope Street, Providence, RI, USA \\ ${ }^{2}$ Professor of Engineering, School of Engineering, Brown University, 182 Hope Street, Providence, RI, USA \\ \{Maria_Restrepo,Brandon_Mayer\}@brown.edu,mundy@lems.brown.edu
}

Keywords: 3-d Object Recognition, 3-d Data Processing, Machine Vision, Bayesian Learning

Abstract: A new representation of 3-d object appearance from video sequences has been developed over the past several years (Pollard and Mundy, 2007, Pollard, 2008, Crispell, 2010), which combines the ideas of background modeling and volumetric multi-view reconstruction. In this representation, Gaussian mixture models for intensity or color are stored in volumetric units. This 3-d probabilistic volume model, PVM, is learned from a video sequence by an on-line Bayesian updating algorithm. To date, the PVM representation has been applied to video image registration (Crispell et al., 2008), change detection (Pollard and Mundy, 2007) and classification of changes as vehicles in 2-d only (Mundy and Ozcanli, 2009. Özcanli and Mundy, 2010). In this paper, the PVM is used to develop novel viewpoint-independent features of object appearance directly in 3-d. The resulting description is then used in a bag-of-features classification algorithm to recognize buildings, houses, parked cars, parked aircraft and parking lots in aerial scenes collected over Providence, Rhode Island, USA. Two approaches to feature description are described and compared: 1) features derived from a PCA analysis of model neighborhoods; and 2) features derived from the coefficients of a 3-d Taylor series expansion within each neighborhood. It is shown that both feature types explain the data with similar accuracy. Finally, the effectiveness of both feature types for recognition is compared for the different categories. Encouraging experimental results demonstrate the descriptive power of the PVM representation for object recognition tasks, promising successful extension to more complex recognition systems

\section{INTRODUCTION AND PRIOR WORK}

A semantic description of $3-\mathrm{d}$ scenes is essential to many urban and surveillance applications. This paper presents a new volumetric representation for the description of 3-d scenes that captures the probabilistic nature of 3-d reconstruction from multiple image views and video sequences. A recognition approach is described to provide semantic labels for aerial scenes including such categories as houses, buildings, parked cars, and parked aircraft. The labels are found by an object classification algorithm based on features extracted directly from a 3-d representation of scene appearance. The resulting object-centered recognition model combines the probability of surface appearance and surface occupancy at densely sampled locations in 3-d space, thus incorporating the ambiguity inherent in surface reconstruction from imagery. To the authors' knowledge, this paper represents the first attempt to base scene classification on a volumetric probabilistic model that learns, in a dense manner, the appearance and geometric information of 3-d scenes from images.

In related work, many 3-d object recognition algorithms have been developed in recent years to search the rapidly growing databases of 3-d models ( $\mathrm{Pa}-$ padakis et al., 2010; Shapira et al., 2010; Drost et al., 2010; Bariya and Nishino, 2010; Bronstein et al., 2011). These recognition algorithms operate on models that are synthetically generated or obtained in a controlled environment using 3-d scanners. Throughout most of the object-retrieval literature, the dominant representation of 3-d geometry is a mesh or point cloud, where the intrinsic properties of the representation are used to describe shape models. However, neither of these representations is able to express the uncertainty and ambiguity of 3-d surfaces inherent in reconstruction from aerial image sequences.

Other recent works have favored volumetric shape descriptors to better cope with isometric deformations (Raviv et al., 2010), and to improve segmentation of 
models into parts and matching of parts from different objects (Shapira et al., 2010). However, the volumetric cues of Raviv and Shapira are defined by an enclosing boundary (represented by a mesh). The probabilistic volume model, PVM, used in this work, learns geometry and appearance in a general framework that can handle changes in viewpoint, illumination and resolution, without regard to surface topology. Therefore, this work addresses the problem of categorizing static objects in scenes learned from images collected under unrestricted conditions, where the only requirement is known camera calibration matrices. It is worth pointing out that the volumetric representation used in this work is different from a representation obtained from a range scanner, not only in that appearance information is stored in the voxels, but also in that surface geometry is estimated in a probabilistic manner from images. The probabilistic framework provides a way to deal with uncertainties and ambiguities that make the problem of computing exact 3-d structures based on 2-d images in general ill-posed (e.g. multiple photo-consistent instances, featureless surfaces, unmodeled appearance variations, and sensor noise).

In another related body of work, image-based recognition in realistic scenes is performed using appearance-based techniques on 2-d image projections. Deformable part models are used (Fergus et al., 2003; Felzenszwalb et al., 2008) to handle shape variations and to account for the random presence and absence of parts caused by occlusion, and variations in viewpoint and illumination. Thomas et al. have extended these ideas to multi-view models, where shape models are based on 2-d descriptors observed in multiple views, and single-view codebooks are learned and interconnected (Thomas et al., 2006). Gupta et al. (Gupta et al., 2009) learn 3-d models of scenes by first recovering the geometry of the scene using a robust structure from motion algorithm, and then transferring 2-d appearance descriptors (SIFT) to the 3 -d points. While the works just mentioned, combine geometry and appearance information to model 3-d scenes, appearance information is only available for a sparse set of 3-d points. The recovered 3-d points correspond to 3-d structures that, when projected onto the 2 -d images yield salient and stable 2-d features. Contrary to the idea of reconstructing 3-d appearance and geometry from a sparse set of 2-d features, the PVM used in this work, models surface occupancy and appearance at every voxel in the scenes. A dense reconstruction of a scene's appearance makes available valuable view-independent characteristics of objects' surfaces that are not captured by sparse 2 -d feature detectors.
In computer vision, local descriptors are widely used in recognition systems developed for 2-d images. Through out the last several years, the vast majority of the local descriptors are obtained using derivative operators on image intensity, e.g. steerable filters (Freeman and Adelson, 1991), HOG (Dalal and Triggs, 2005) and SIFT (Lowe, 2004). Inspired by the success of local descriptors in feature-based 2-d recognition, the work presented in this paper uses local descriptors for 3-d recognition in volumetric probabilistic models. In contrast to a mesh representation, where derivatives are only approximately defined for arbitrary topologies, the information stored at each voxel (to be defined later), allows for a natural way to perform dense differential operations. In this work, derivatives are computed using 3 -d operators that are based on a second degree Taylor series approximation of the volumetric appearance function to be defined in a later section. The performance of the Taylor-based features and features extracted from the PCA analysis of the same volumetric feature domain, are compared. It is shown that both features have comparable descriptive power and recognition accuracy, providing an avenue to generalizing the methods that have been used successfully in 2-d derivative-based recognition systems to the 3 -d probabilistic volume models used in this work.

To date, the PVM has been been applied to video image registration (Crispell et al., 2008), change detection in images (Pollard and Mundy, 2007) and classification of changes in satellite images as vehicles (Mundy and Ozcanli, 2009: Özcanli and Mundy, 2010). For the purpose of these applications, a small number of probabilistic volume models are needed. However, in order to perform multi-class object recognition experiments it was necessary to train a larger number of models. The aerial imagery was collected in Providence, RI, USA, and used to learn 18 volumetric models. These models represent a variety of landscapes and contain large number of objects per scene. Each scene model, composed of approximately 30 million voxels, covers an estimate ground area of $(500 \times 500) \mathrm{m}^{2}$. The areal video data, camera matrices and other supplemental material are available on line 1

In summary, the contributions of the work presented in this paper are:

1. To be the first work to perform object categorization task on probabilistic volume models that learn geometry and appearance information everywhere in space, and that are learned in unrestricted settings from images sequences.

\footnotetext{
${ }^{1}$ http://vision.lems.brown.edu/project_desc/ Object-Recognition-in-Probabilistic-3D-Scenes
} 


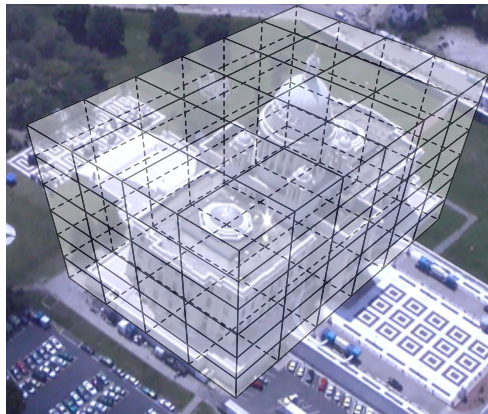

(a)

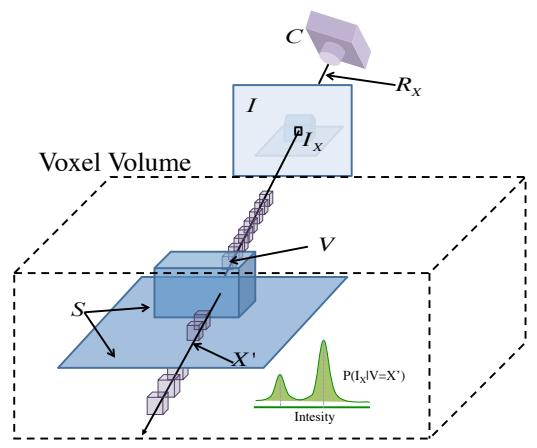

(b)

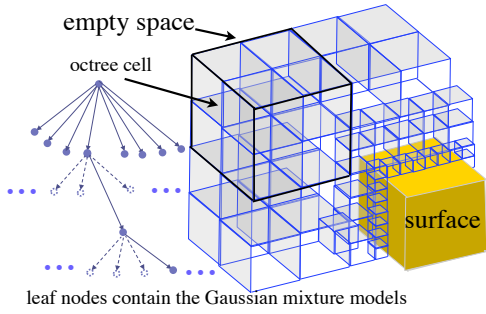

(c)

Figure 1: 1a and 1b PVM proposed by Pollard and Mundy (Pollard and Mundy, 2007; Pollard, 2008). 1b explains the voxel notation, a pixel $I_{X}$ back projects into a ray of voxels $R_{X}, V$ is the unique voxel along $R_{X}$ that produces the intensity $I_{X}$. $1 \mathrm{c}$ Octree subdivision of space proposed by Crispell (Crispell, 2010).

2. To characterize for the first time the local, 3-d information in the PVM. The result are novel, view-invariant, volumetric features that describe local neighborhoods of the probabilistic information of 3-d surface geometry and appearance in the scenes.

3. A demonstration of the descriptive power, through rigorous analysis of function approximation and object recognition experiments, of features based on a Taylor series approximation, and PCA analysis of the probabilistic information in the models. Encouraging initial recognition results promise successful extensions based on generalization of 2-d features e.g. Harris corners (Harris and Stephens, 1988), HOG (Dalal and Triggs, 2005), SIFT (Lowe, 2004), and 3-d differential features (Sipiran and Bustos, 2010, Raviv et al., 2010), to the probabilistic models in question.

4. The creation of the largest database of probabilistic volume models available today.

The rest of the paper is organized as follows: Section 1.1 explains the probabilistic volume model used to learn the 18 areal sites of the city of Providence used in this work. Section 2 discusses two types of features used to model local neighborhoods in the volumetric scenes. Section 3 explains category learning and object classification. Section 4 presents the experimental results. Finally, conclusions and further work are described in Sections 5 and 6 ,

\subsection{Probabilistic Volume Model}

Pollard and Mundy (2007) proposed a probabilistic volume model that can represent the ambiguity and uncertainty in 3-d models derived from multiple image views. In Pollard's model (Pollard and Mundy,
2007; Pollard, 2008), a region of three-dimensional space is decomposed into a regular 3-d grid of cells, called voxels (See Figure 1). A voxel stores two kinds of state information: (i) the probability that the voxel contains a surface element and (ii) a mixture of Gaussians that models the surface appearance of the voxel as learned from a sequence of images. The surface probability is updated by incremental Bayesian learning (see Equation 1 below), where the probability of a voxel $\mathrm{X}$ containing a surface element after $\mathrm{N}+1 \mathrm{im}$ ages increases if the Gaussian mixture (see Equation 2 below) at that voxel explains the intensity observed in the $\mathrm{N}+1$ image better than any other voxel along the projection ray. The resulting models look more like volumetric models obtained from CT scans than models obtained from point clouds generated by range scanners (see Figure 2).

$$
\begin{array}{r}
P^{N+1}(X \in S)=P^{N}(X \in S) \frac{p^{N}\left(I_{X}^{N+1} \mid X \in S\right)}{p^{N}\left(I_{X}^{N+1}\right)} \\
p(I)=\sum_{k=1}^{3} \frac{w_{k}}{W}\left(\frac{1}{\sqrt{2 \pi \sigma_{k}^{2}}} \exp ^{-\frac{\left(I-\mu_{k}\right)^{2}}{2 \sigma_{k}^{2}}}\right)
\end{array}
$$

In a fixed-grid voxel representation, most of the voxels may correspond to empty areas of a scene, making storage of large, high-resolution scenes prohibitively expensive. Crispell (2010) proposed a continuously varying probabilistic scene model that generalizes the discrete model proposed by Pollard and Mundy. Crispell's model allows non-uniform sampling of the volume leading to an octree representation that is more space-efficient and can handle finer resolution required near 3-d surfaces, see Figure 1c

The octree representation (Crispell, 2010), makes it feasible to store models of large urban areas. However, learning times of large scenes using the PVM 

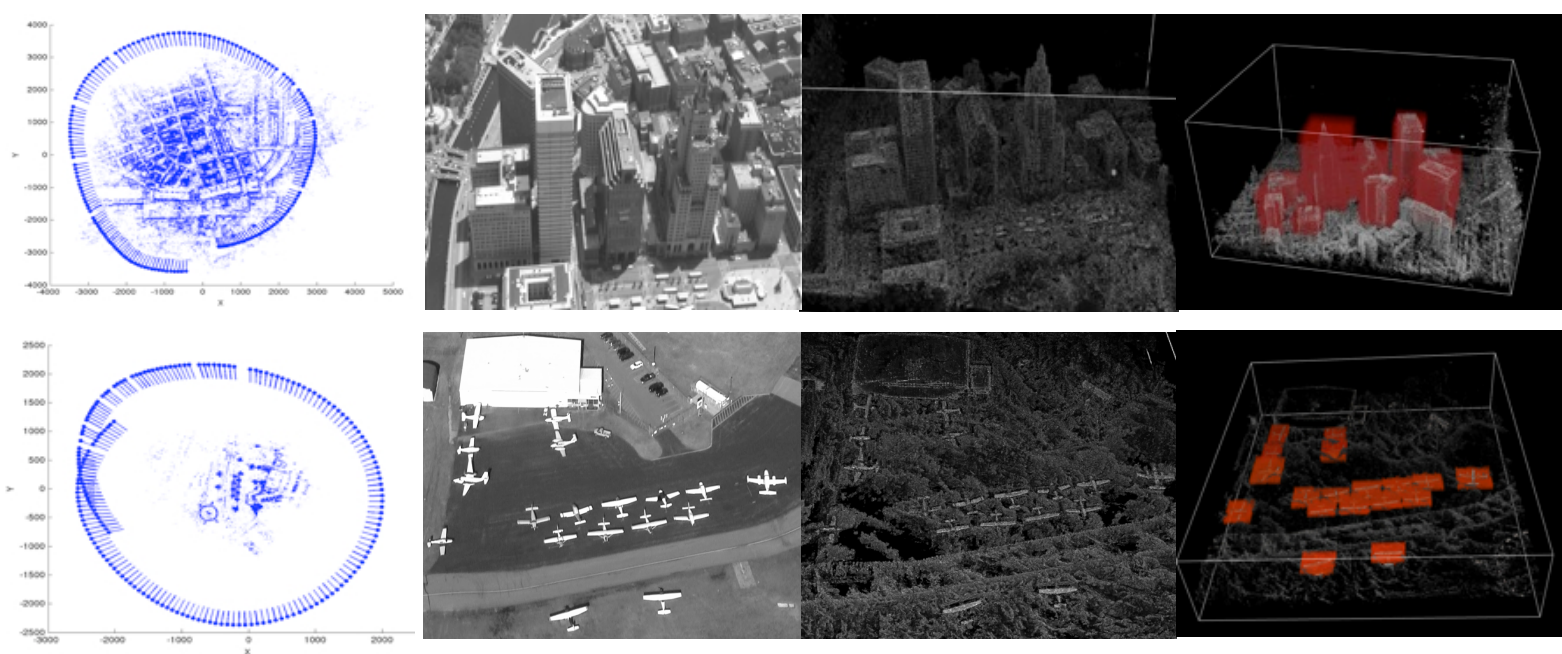

Figure 2: From left to right (column by column): Camera path and 3-d points (only used for visualization purposes) obtained using Bundler (Snavely and Seitz, 2006). Details of collected video frames. The learned expected appearance volumes, EVM. Examples of bounding boxes around objects of interest (this figure is best seen in color).

remained impractical until recently, when a GPU implementation was developed by Miller et al. (2011). Training times decrease by several orders of magnitudes, depending on available computing resources and model complexity, see (Miller et al., 2011) for performance comparisons evaluated on single core CPU and OpenCL implementation on CPU and GPU. With a GPU framework in place is now feasible to think of multi-class object recognition tasks where large number of objects are required for training.

\section{VIEW INDEPENDENT 3-D FEATURES}

This section describes how the geometry and appearance information at every voxel are used to compute a voxel's expected appearance. It then describes two approaches used to characterize the local information (i.e. expected appearances) in the volumetric scenes, namely PCA features and Taylor features.

\subsection{Expected Volume Model}

Though the work by Pollard was designed to detect changes in a new image, the occupancy and appearance information can be used to render synthetic images of the expected scene appearance (Pollard, 2008). For every pixel in the image, its intensity is the summation, across all voxels in its projection ray, of the expected color of the voxel and the likelihood of that voxel containing a surface element and it not being occluded. Consider a pixel $I_{X}$, which back projects into a ray of voxels $R_{X}$, if $V$ is the unique voxel along $R_{X}$ that causes the intensity value at the pixel, then the expected intensity at $I_{X}$ is explained by (3) and (4) (also see Figure 1b).

$$
\begin{array}{r}
E\left(I_{X}\right)=\sum_{X^{\prime} \in R_{X}} E\left(I_{X} \mid V=X^{\prime}\right) P\left(V=X^{\prime}\right) \\
=\sum_{X^{\prime} \in R_{X}} E\left(I_{X} \mid V=X^{\prime}\right) P\left(X^{\prime} \in S\right) P\left(X^{\prime} \text { is not occluded }\right)
\end{array}
$$$$
E\left(I_{X} \mid V=X^{\prime}\right) \text { represents the expected intensity, }
$$
given that voxel $X^{\prime} \in R_{X}$ produced the intensity seen in the image. This quantity is obtained from the mixture of Gaussians stored at voxel $X^{\prime} . P\left(X^{\prime} \in S\right)$ is the probability of $X^{\prime}$ containing a surface element and it is also stored at $X^{\prime} . P\left(X^{\prime}\right.$ is not occluded $)$ is defined as the probability that all voxels (along $R_{X}$ ) between $X^{\prime}$ and the camera contain empty space i.e. $P\left(X^{\prime}\right.$ is not occluded $)=\prod_{X^{\prime \prime}<X^{\prime}}\left(1-P\left(X^{\prime \prime} \in S\right)\right)$.

For every ray containing a particular voxel $X^{\prime}$, the quantity $E\left(I_{X} \mid V=X^{\prime}\right) P\left(X^{\prime} \in S\right)$ remains unchanged, and the only ray-dependent term is $P\left(X^{\prime}\right.$ is not occluded $)$. When learning neighborhood configurations in the PVM, only the ray-independent information is taken into account. The information at every voxel is combined into to the quantity in Equation (5) (see below), here referred to as a voxel's expected appearance, and the volume of expected appearances, as the expectation volume model, EVM.

$$
E\left(I_{x} \mid V=X^{\prime}\right) P\left(X^{\prime} \in S\right)
$$

\subsection{PCA Features}

One way to represent the volumetric model is by identifying local spatial configurations that account for 
most of the variation in the data. Principal Component Analysis (PCA) is carried out to find the orthonormal basis that represents the volumetric samples in the best mean squared error sense. The principal components are arranged in decreasing order of variation as given by the eigenvalues of the sample scatter matrix.

In order to perform PCA, feature vectors are obtained by sampling locations on the scene according to the octree structure, i.e. fine sampling in regions near surfaces and sparse sampling of empty space. At each sampled location, $n_{x} \hat{l} \times n_{y} \hat{l} \times n_{z} \hat{l}$ cubical regions are extracted (centered at the sampled location), where $\hat{l}$ is the length of the smallest voxel present in the 3 - $d$ scene. The extracted regions are arranged into vectors by traversing the space at a resolution of $\hat{l}$, and using a raster visitation schedule.

The scatter matrix $\mathbf{S}$, of randomly sampled vectors, is updated using a parallel scheme (Chan et al., 1979) to speed up computation, and the principal components are found by the eigenvalue decomposition of $\mathbf{S}$. In the PCA space, every neighborhood (represented by a $d$-dimensional feature vector $\mathbf{x}$ ) can be exactly expressed as $\mathbf{x}=\overline{\mathbf{x}}+\sum_{i=1}^{d} a_{i} \mathbf{e}_{i}$, where $\mathbf{e}_{i}$ are principal axes associated with the $d$ eigenvalues, and $a_{i}$ are the corresponding coefficients. A kdimensional $(k<d)$ approximation of the neighborhoods can be obtained by using the first $k$ principal components i.e. $\tilde{\mathbf{x}}=\overline{\mathbf{x}}+\sum_{i=1}^{k} a_{i} \mathbf{e}_{i}$. Section 4 presents a detailed analysis of the reconstruction error of local neighborhoods, namely $|\mathbf{x}-\tilde{\mathbf{x}}|^{2}$, as a function of dimension and training set size. In the remainder of this paper, the vector arrangement of projection coefficients in the PCA space is referred as a PCA feature.

\subsection{Taylor Features}

Mathematically, the appearance function in the scene can be approximated (locally) by its Taylor series expansion. The computation of derivatives in the expectation volume model, EVM, can be expressed as a least square error minimization of the following energy function.

$$
E=\sum_{i=-n i}^{n i} \sum_{j=-n j}^{n j} \sum_{k=-n k}^{n k}(V(i, j, k)-\tilde{V}(i, j, k))^{2}
$$

Where $\tilde{V}(i, j, k)$ is the Taylor series approximation of the expected 3-d appearance of a volume $V$ centered on the 3 -d point $(i, j, k)$. Using the second degree Taylor expansion of $V$ about $(0,0,0)$, 6 becomes

$$
E=\sum_{\mathbf{x}}\left(V(\mathbf{x})-V_{0}-\mathbf{x}^{\mathbf{T}} \mathrm{G}-\frac{1}{2 !} \mathbf{x}^{\mathbf{T}} \mathrm{H} \mathbf{x}\right)^{2}
$$

Where $V_{0}, \mathrm{G}, \mathrm{H}$ are the zeroth derivative, the gradient vector and the Hessian matrix of the volume of expected 3-d appearance about the point $(0,0,0)$, respectively. The coefficients for 3-d derivative operators can be found by minimizing (7) with respect to the zeroth, first and second order derivatives. The computed derivative operators are applied algebraically to neighborhoods in the EVM. The responses to the 10 Taylor operators, which correspond to the magnitude of the zeroth, first and second order derivatives, are arranged into 10-dimensional vectors and are referred to as Taylor features.

\section{3-D OBJECT LEARNING AND RECOGNITION}

This section explains in detail the model used to learn five object categories. It is important to keep in mind that models are based on either Taylor features of PCA features, but not both. The results obtained for the two representations are presented in Section 4

\subsection{The Model: Bag of Features}

Bag-of-features models have their origins in texture recognition (Varma and Zisserman, 2009; Leung and Malik, 1999) and bag-of-word representations for text categorization (Joachims, 1997). Their application to categorization of visual data is very popular in the computer vision community (Sivic et al., 2005, Csurka et al., 2004) and have produced impressive results in benchmark databases (Zhang et al., 2007). The independence assumptions inherent to bag-offeatures representation make learning models for few object categories a simple task, assuming enough training samples are available to learn the classification space. In this paper, a bag-of-features representation is constructed for five categories as outlined in the following subsections.

\subsection{Learning a Visual Vocabulary with $k$-means}

In order to produce a finite dictionary of 3-d expected appearance patterns, the scenes are represented by a set of descriptors (Taylor or PCA) that are quantized using k-means-type clustering. Two major limitations of k-means clustering must be overcome: (i) the algorithm does not determine the best number of means, i.e $k$, and (ii) it converges to a local minimum that may not represent the optimum placement of cluster centers. 
To address (i), there exist available algorithms to automatically determine the number of clusters (Pelleg and Moore, 2000, Hamerly and Elkan, 2003). However, in the experiments in this paper the algorithm was run using various values of $k$. An optimal value was selected based on object classification performance and running times. Disadvantage (ii) requires careful attention because the success of k-means depends substantially on the starting positions of the means. In the experiments, the training scenes are represented by millions of descriptors (even if only a percentage of them are used), and random sampling of $k$ means, where $k<<1 \times 10^{6}$, may not provide a good representation of the 3 -d appearance patterns.

The means are initialized using the algorithm proposed by Bradley and Fayyad (1998), which has been shown to perform well for large data sets (Maitra et al., 2010). In the initialization algorithm (Bradley and Fayyad, 1998), a random set of sub-samples of the data is chosen and clustered via modified $\mathrm{k}$-means. The clustering solutions are then clustered using classical k-means, and the solution that minimizes the sum of square distances between the points and the centers is chosen as the initial set of means. In order to keep computation time manageable, while still choosing an appropriate number of sub-samples (10 being suggested in (Maitra et al., 2010, Bradley and Fayyad, 1998)), an accelerated k-means algorithm (Elkan, 2003) is used whenever the classical k-means procedure is required.

The large number of volumetric training features can only be practically processed using parallel computation. While parallel clustering algorithms are available (Judd et al., 1998), message passing between iterations could not be easily implemented for the current framework. Therefore, an approximate kmeans method was selected, which is a modification of the refinement algorithm by Bradley and Fayyad (1998). The modified k-means algorithm is the following:

1. Sample an initial set of means, $S P$, as described above

2. Divide training samples into J blocks. Let $C M=\emptyset$

3. Process each block in parallel as follows:

a. Let $S_{i}$ be the data in block $J_{i}$

b. $C M_{i}=$ AcceleratedKMeans $\left(S P, S_{i}, K\right)$

4. $C M=\bigcup_{i=0}^{j} C M_{i}, F M=\emptyset$

5. Process each $C M_{i}$ in parallel as follows: a. $F M_{i}=$ AcceleratedKMeans $\left(C M_{i}, C M, K\right)$

6. $F M=\underset{F M_{i}}{\arg \min } \operatorname{Distortion}\left(F M_{i}, C M\right)$

The minimization function, Distortion $\left(F M_{i}, C M\right)$, computes the sum of square distances of each data point to its nearest mean (for all $\mathrm{J}$ estimates). The set of clusters with the smallest distortion value is cho- sen as the final solution, $F M$. The proposed algorithm does not seek to improve the complexity of the traditional k-means algorithm but to manage memory requirements and allow parallel processing of large data sets.

\subsection{Learning and Classification}

With a 3-d appearance vocabulary in place, individual objects are represented by feature vectors that arise from the quantization of the PCA or Taylor descriptors present in that object. These feature vectors can be used in supervised multi-class learning, where a naive Bayes classifier is used for its simplicity and speed. During learning, the classifier is passed training objects used to adjust the decision boundaries; during classification, the class label with the maximum a posteriori probability is chosen to minimize the probability of error.

Formally, let the objects of a particular category be the set $\mathbf{O}_{l}=\bigcup_{i=1}^{N_{l}} \mathbf{o}_{i}$, where $l$ is the class label and $N_{l}$ is the number of objects with class label $l$. Then, the set of all labeled objects is defined as $\mathbf{O}=\bigcup_{l=1}^{N_{c}} \mathbf{O}_{l}$, where $N_{c}$ is the number of categories. Let the vocabulary of 3-d expected appearance patterns be defined as $\mathbf{V}=$ $\bigcup_{i=1}^{k} \mathbf{v}_{i}$, where $k$ is the number of cluster centers in the vocabulary. From the quantization step a count is obtained, $c_{i j}$, of the number of times a cluster center, $\mathbf{v}_{i}$, occurs in object $\mathbf{o}_{j}$. Using Bayes formula, the $a$ posteriori class probability is given by:

$$
P\left(C_{l} \mid \mathbf{o}_{i}\right) \propto P\left(\mathbf{o}_{i} \mid C_{l}\right) P\left(C_{l}\right)
$$

The likelihood of an object is given by the product of the likelihoods of the independent entries of the vocabulary, $P\left(\mathbf{v}_{j} \mid C_{l}\right)$, which are estimated during learning. The full expression for the class posterior becomes:

$$
\begin{aligned}
P\left(C_{l} \mid \mathbf{o}_{i}\right) & \propto P\left(C_{l}\right) \prod_{j=1}^{k} P\left(\mathbf{v}_{j} \mid C_{l}\right)^{c_{j i}} \\
& \propto P\left(C_{l}\right) \prod_{j=1}^{k}\left(\frac{\sum_{m=1: \mathbf{o}_{m} \in \mathbf{O}_{l}}^{N_{m}} c_{j m}}{\sum_{n=1}^{k} \sum_{m=1: \mathbf{o}_{m} \in \mathbf{o}_{l}}^{N_{m}} c_{n m}}\right)^{c_{j i}}
\end{aligned}
$$

According to the Bayes decision rule, every object is assigned the label of the class with the largest a posteriori probability. In practice, log likelihoods were computed to avoid underflow of floating point computations. 


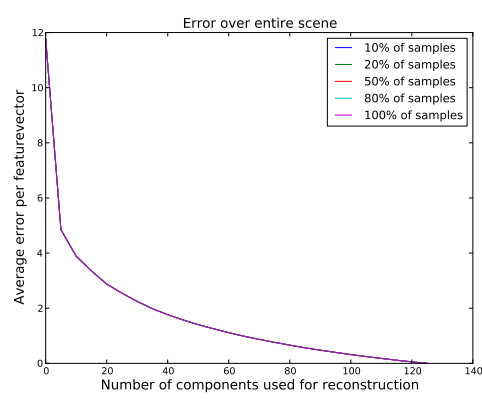

(a)

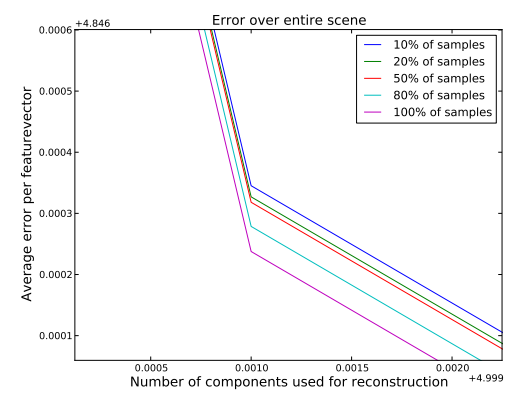

(b)

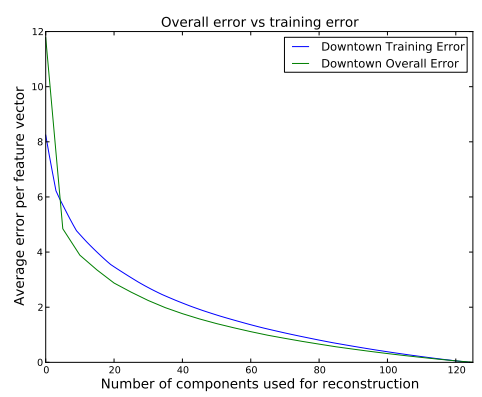

(c)

Figure 3: Analysis of reconstruction error. 3a Error as a function of the number of components used. The different curves represent different proportion of samples used to perform PCA. 3b A zoomed-in detail of 3a 3c Comparison of errors obtained over training neighborhoods vs. all available neighborhoods.

\section{EXPERIMENTS AND RESULTS}

The data collection and scene reconstruction processes are now described, followed by comparisons of scene data modeling accuracy based on either PCA or Taylor features. The section concludes with multiclass object recognition results, where objects from 8 scenes were classified among 5 categories; planes, cars, houses, buildings, and parking lots. Training samples consist of labeled objects from 10 scenes (different from the ones used for testing). In order to localize surface features through k-means, only features centered at leaf-cells at the finest resolution level of the octree were considered i.e. cells containing high occupancy probability

\subsection{Data Collection and Scene Formation}

The aerial data used to build 18 different probabilistic volume scenes was collected from a helicopter flying over Providence, RI, USA, and its surroundings. An approximate resolution of $30 \mathrm{~cm} /$ pixel was obtained in the imagery and translated to $30 \mathrm{~cm} / \mathrm{voxel}$ in the models. The camera matrices for all image sequences were obtained using Bundler (Snavely and Seitz, 2006). The probabilistic volume models were learned using a GPU implementation (Miller et al., 2011). For multi-class object recognition, bounding boxes around objects of interest were given a class label. Ten scenes were used for training and eight for testing. Figure 2 contains examples of aerial images collected for these experiments, the EVMs and the bounding boxes used to label objects of interest.

\subsection{Neighborhood Reconstruction Error}

Ideally, the difference between the original expected appearance data and the data approximated using PCA or a Taylor series expansion should be small. The difference between the reconstructed data and the original data was measured as the average square difference between neighborhoods, i.e. $\frac{1}{N} \sum_{i=1}^{N_{\text {train }}}|\mathbf{x}-\hat{\mathbf{x}}|^{2}$, where $\mathbf{x}$ and $\hat{\mathbf{x}}$ are the vector arrangement of the original and the approximation neighborhoods, respectively. For Taylor features, $\hat{\mathbf{x}}=V_{0}+\mathbf{x}^{\mathbf{T}} \mathrm{G}+\frac{1}{2 !} \mathbf{x}^{\mathbf{T}} \mathrm{Hx}$. For PCA features $\hat{\mathbf{x}}=\overline{\mathbf{x}}+\sum_{i=1}^{10} a_{i} \mathbf{e}_{i}$. In the experiments, the size of the extracted neighborhoods was $5 \hat{l} \times 5 \hat{l} \times 5 \hat{l}, \hat{l}$ being the length of the smallest voxel in the model. The error was computed for the top scene in Figure 2, here referred to as the Downtown scene.

Using all available neighborhoods to learn the PCA basis is impractical; thus, a set of experiments were performed to evaluate the reconstruction error for different sizes of randomly chosen neighborhoods. Figures $3 \mathrm{a}$ and $3 \mathrm{~b}$ show the reconstruction error for different sample sizes (as a percentage of the total number of neighborhoods). The error was basically identical for all computed fractions and $10 \%$ was the fraction used for the remaining of the experiments. Figure $3 \mathrm{c}$ compares the projection error over the training samples and the overall projection error (over all available neighborhoods). The curves are very similar, indicating that the learned basis represents the training and testing data with comparable accuracy. Finally, the reconstruction error for a 10-dimensional approximation in the PCA space was compared to the reconstruction error achieved using a $2^{\text {nd }}$-degree Taylor approximation. The results in Table 11 indicate that a $2^{\text {nd }}$-degree Taylor approximation represents expected appearance of 3-d patterns with only slight less accuracy than a PCA projection onto a 10dimensional space. 
Table 1: Average approximation error over all $5 \times 5 \times 5$ neighborhoods. PCA and Taylor approximations are compared for the Downtown scene.

\begin{tabular}{|c|c|c|}
\hline Scene Name & PCA Error & Taylor Error \\
\hline Downtown & 3.88 & 4.05 \\
\hline
\end{tabular}

\subsection{3-d Object Recognition}

This section presents multi-class object recognition results. Five object categories were learned: planes, cars, buildings, houses, and parking lots. Table 2 presents the number of objects in each category used during training and classification.

Table 2: Number of objects in every category.

\begin{tabular}{|c|c|c|c|c|c|}
\hline & Planes & Cars & Houses & Buildings & Parking Lots \\
\hline Train & 18 & 54 & 61 & 24 & 27 \\
\hline Test & 16 & 29 & 45 & 15 & 17 \\
\hline
\end{tabular}

Two measurements were used to evaluate the classification performance: (i) classifier accuracy (i.e the fraction of correctly classified objects), and (ii) the confusion matrix. During classification experiments, the number of clusters in the codebook was varied from $k=2$ to $k=100$. Figure 4 presents classification accuracy as a function of the number of clusters. For both, Taylor-based features and PCA-based features, the performance improves rapidly up to a 20 -word codebook, with little or no improvement for larger vocabularies. Thus, for the remaining of the experiments $k$ was set to 20 .

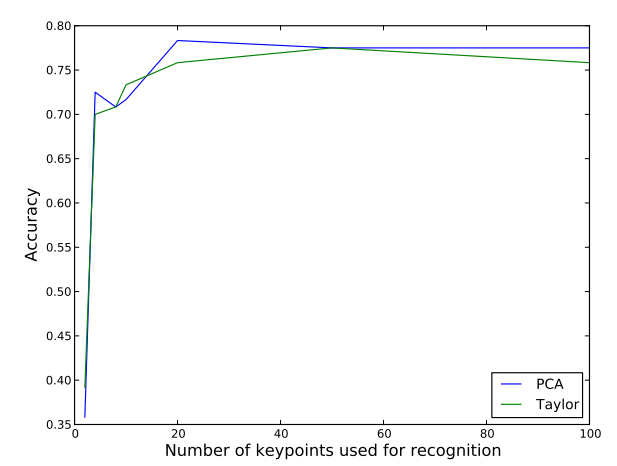

Figure 4: Classification Accuracy. The curves represent the fraction of correctly classified objects as a function of the number of clusters.

Figure 5 presents examples of class distributions learned with PCA and Taylor codebooks of twenty features. To facilitate interpretation, the volumetric form of the vocabulary entries are arranged along the $\mathrm{X}$-axis. It is important to keep in mind that each voxel (in the volumetric feature), contains its expected 3d appearance as defined by Eq. 5. The value of expected 3-d appearance ranges from $[0,2]$ (and the color used in the volumetric rendering from black to white respectively). For empty space, the information in the voxels is dominated by the occupancy probability, which takes values in the interval $[0,1]$; thus, empty neighborhoods appear black. Appearance values, which are initially learned between $[0,1]$, are offset to $[1,2]$, to avoid confusing dark surfaces with empty space. White voxels represent white surfaces with a high occupancy probability; dark surfaces are represented by gray voxels. For the planes category, see Figures 5a and 5c, empty neighborhoods, white surface neighborhoods and neighborhoods containing half white-surface space and half empty space are the most common features. On the other hand, the buildings category, see Figures $5 \mathrm{~b}$ and $5 \mathrm{~d}$, is represented by mid range neighborhoods corresponding to dark surfaces and slowly changing derivatives.

Finally, the confusion matrices for a 20-keyword vocabulary of PCA-based features and Taylor-based features, are shown in Tables 6a and 6b. Both methods recognize planes, cars and parking lots with high accuracy. Lower performance for buildings and houses is expected, since a more discriminative model is needed to successfully differentiate such similar categories. The PCA-based representation is slightly better at learning effective models for cars than the Taylor based representation.

\section{CONCLUSION}

This paper presented a completely new representation for object recognition models, where view-invariant features were extracted directly from 3 -d probabilistic information. The representation was used to learn and recognize objects from five different categories. To the author's knowledge, this work represents the first attempt to apply this representation to the classification of aerial scenes or indeed any type of scene. The performance of the proposed features, was rigorously tested through reconstruction accuracy and object categorization experiments. The recognition results are very encouraging with high accuracy on labeling bounded regions containing objects of the selected categories. The experiments show that differential geometry features derived from appearance lead to essentially the same recognition performance as PCA. This suggests that additional features representing geometric relationships defined on differential geometry are likely to have good performance and represent a basis for formally extending the feature vocabulary.

While there are many computational and storage challenges faced when learning multiple object cate- 


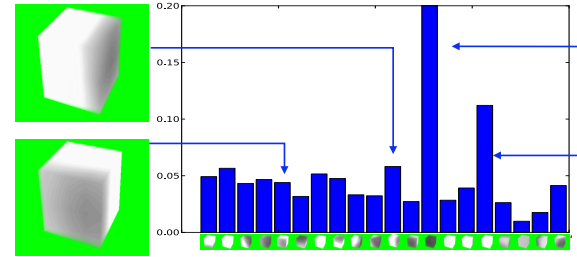

(a) Plane - PCA
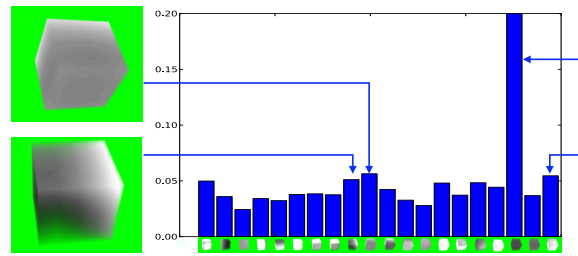

(c) Plane - Taylor
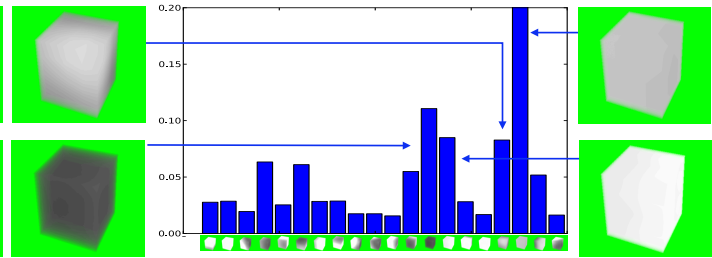

(b) Building - PCA
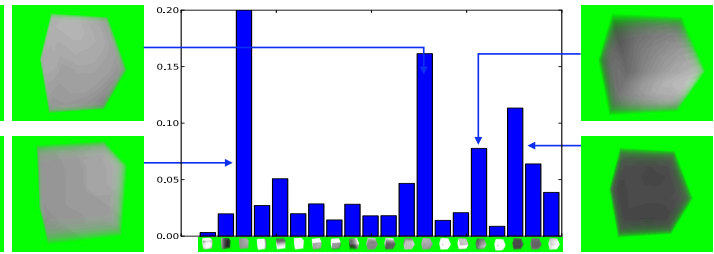

(d) Building - Taylor

Figure 5: Class histograms for plane and building categories. The top row corresponds to class representations learned with PCA-based features. The bottom row corresponds to those learned with Taylor-based features. The x-axis shows the volumetric form of the 20 features; the $y$-axis, the probability of each feature. The most probable volumetric features for each class are shown beside each histogram.

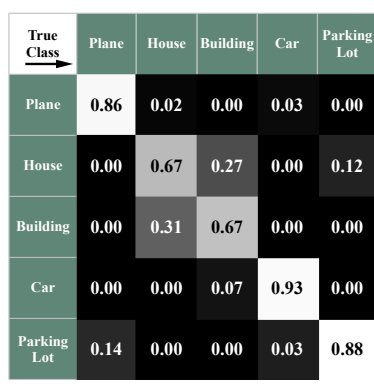

(a) PCA

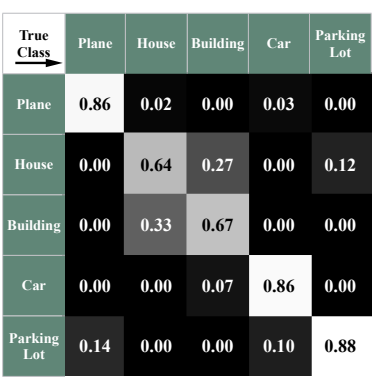

(b) Taylor
Figure 6: Confusion matrix for a 20-keyword codebook of PCA based features on the left and Taylor based features on the right.

gories in large volumetric scenes, the work presented in this paper makes an important contribution towards true 3-d, view-independent object recognition. The object categorization results demonstrate the descriptive power of the PVM for 3-d object recognition and open an avenue to more complex recognition systems for dense, 3-d probabilistic scenes.

\section{FURTHER WORK}

The current feature representation will be extended to incorporate features detected with differential operators that have been used successfully in 2$\mathrm{d}$ featured-based recognition systems and 3-d object retrieval algorithms, e.g. 2-d and 3-d features based on the Harris operator (Harris and Stephens, 1988, Sipiran and Bustos, 2010), 3-d heat kernels based on the Laplace-Beltrami operator (Raviv et al., 2010),
SIFT features (Lowe, 2004), HOG features (Dalal and Triggs, 2005) and others. The occlusion, shadows and 3-d relief present in the imagery collected for the experiments presented in this work, pose great challenges to 2-d multi-view recognition systems. However, in future work, it will prove informative to compare 2-d multi-view systems to the framework presented in this paper.

The probabilistic scenes learned for this work have known orientation and scale. In order to keep feature-base representation of objects compact, future work will explore representations for scale-invariant and isometric features. Localization of objects is also a desirable goal for future algorithms.

Finally, more advanced recognition models should make full use of the geometric relations inherent in the probabilistic volume model. Compositional recognition models could represent a venue to learn and share parts, allowing for object representations that are efficient, discriminative and geometrically coherent.

\section{REFERENCES}

Bariya, P. and Nishino, K. (2010). Scale-Hierarchical 3D Object Recognition in Cluttered Scenes. IEEE Conference on Computer Vision and Pattern Recognition.

Bradley, P. S. and Fayyad, U. M. (1998). Refining Initial Points for K-Means Clustering. In Proceedings of the 15th International Conference on Machine Learning.

Bronstein, A. M., Broinstein, M. M., Guibas, L. J., and Ovsjanikov, M. (2011). Shape Google: Geometric Words 
and Expressions for Invariant Shape Retrieval. $A C M$ Transactions on Graphics.

Chan, T. F., Golub, G. H., and LeVeque, R. J. (1979). Updating Formulae and a Pairwise Algorithm for Computing Sample Variances. Technical report, Department of Computer Science. Stanford University.

Crispell, D., Mundy, J., and Taubin, G. (2008). ParallaxFree Registration of Aerial Video. In BMVC.

Crispell, D. E. (2010). A Continuous Probabilistic Scene Model for Aerial Imagery. PhD thesis, School of Engineering, Brown University.

Csurka, G., Dance, C. R., Fan, L., Willamowski, J., and Bray, C. (2004). Visual categorization with bags of keypoints. In In Workshop on Statistical Learning in Computer Vision, ECCV.

Dalal, N. and Triggs, B. (2005). Histograms of oriented gradients for human detection. In IEEE Conference on Computer Vision and Pattern Recognition.

Drost, B., Ulrich, M., Navab, N., and Ilic, S. (2010). Model Globally, Match Locally: Efficient and Robust 3D Object Recognition. IEEE Conference on Computer Vision and Pattern Recognition.

Elkan, C. (2003). Using the triangle inequality to accelerate k-means. In Proceedings of the Twentieth International Conference on Machine Learning.

Felzenszwalb, P., McAllester, D., and Ramanan, D. (2008). A discriminatively trained, multiscale, deformable part model. In IEEE Conference on Computer Vision and Pattern Recognition.

Fergus, R., Perona, P., and Zisserman, A. (2003). Object class recognition by unsupervised scale-invariant learning. In IEEE Conference on Computer Vision and Pattern Recognition.

Freeman, W. and Adelson, E. (1991). The design and use of steerable filters. IEEE Transactions on Pattern Analysis and Machine Intelligence.

Gupta, P., Arrabolu, S., Brown, M., and Savarese, S. (2009). Video scene categorization by 3D hierarchical histogram matching. In International Conference on Computer Vision.

Hamerly, G. and Elkan, C. (2003). Learning the k in kmeans. In Seventeenth annual conference on neural information processing systems (NIPS).

Harris, C. and Stephens, M. (1988). A combined corner and edge detector. Alvey vision conference.

Joachims, T. (1997). Text Categorization with Support Vector Machines: Learning with Many Relevant Features. In $E C M L$.

Judd, D., McKinley, P., and Jain, A. (1998). Large-scale parallel data clustering. IEEE Transactions on Pattern Analysis and Machine Intelligence.

Leung, T. and Malik, J. (1999). Recognizing surfaces using three-dimensional texton. In Proceedings of the Seventh IEEE International Conference on Computer Vision.

Lowe, D. G. (2004). Distinctive Image Features from ScaleInvariant Keypoints. International Journal of Computer Vision.
Maitra, R., Peterson, A. D., and Ghosh, A. P. (2010). A systematic evaluation of different methods for initializing the K-means clustering algorithm. In IEEE Transactions of Knowledge and Data Engineering.

Miller, A., Jain, V., and Mundy, J. (2011). Real-time Rendering and Dynamic Updating of 3-d Volumetric Data. In Proceedings of the Fourth Workshop on General Purpose Processing on Graphics Processing Units.

Mundy, J. L. and Ozcanli, O. C. (2009). Uncertain geometry: a new approach to modeling for recognition. In 2009 SPIE Defense, Security and Sensing Conference.

Özcanli, O. and Mundy, J. (2010). Vehicle Recognition as Changes in Satellite Imagery. In International Conference on Pattern Recognition.

Papadakis, P., Pratikakis, I., and Theoharis, T. (2010). PANORAMA: A 3D Shape Descriptor Based on Panoramic Views for Unsupervised 3D Object Retrieval. International Journal of Computer Vision.

Pelleg, D. and Moore, A. (2000). X-means: Extending Kmeans with $\mathrm{E}$ cient Estimation of the Number of Clusters. In International Conference on Machine Learning.

Pollard, T. (2008). Comprehensive 3-d Change Detection Using Volumetric Appearance Modeling. $\mathrm{PhD}$ thesis, Division of Applied Mathematics, Brown University.

Pollard, T. and Mundy, J. (2007). Change Detection in a 3-d World. In IEEE Conference on Computer Vision and Pattern Recognition.

Raviv, D., Bronstein, M. M., Bronstein, A. M., and Kimmel, R. (2010). Volumetric heat kernel signatures. In 3DOR '10: Proceedings of the ACM workshop on $3 D$ object retrieval.

Shapira, L., Shalom, S., Shamir, A., Cohen-Or, D., and Zhang, H. (2010). Contextual Part Analogies in 3D Objects. International Journal of Computer Vision.

Sipiran, I. and Bustos, B. (2010). A Robust 3D Interest Points Detector Based on Harris Operator. In Eurographics Workshop on 3D Object Retrieval.

Sivic, J., Russell, B., Efros, A., Zisserman, A., and Freeman, W. (2005). Discovering objects and their location in images. In International Conference on Computer Vision.

Snavely, N. and Seitz, S. (2006). Photo tourism: exploring photo collections in 3D. ACM Transactions on Graphics.

Thomas, A., Ferrar, V., Leibe, B., Tuytelaars, T., Schiel, B., and Van Gool, L. (2006). Towards Multi-View Object Class Detection. In IEEE Conference on Computer Vision and Pattern Recognition.

Varma, M. and Zisserman, A. (2009). A Statistical Approach to Material Classification Using Image Patch Exemplars. IEEE Transactions on Pattern Analysis and Machine Intelligence.

Zhang, J., Marszalek, M., Lazebnik, S., and Schmid, C. (2007). Local Features and Kernels for Classification of Texture and Object Categories: A Comprehensive Study. Int. J. Comput. Vision. 